\title{
Promoção da saúde da mulher brasileira e a alimentação saudável: vozes e discursos evidenciados pela Folha de SP
}

\author{
The promotion of health of the Brazilian female and healthy eating: \\ discourses and opinions printed in the Brazilian newspaper \\ Folha de São Paulo
}

\author{
Mariella Silva de Oliveira-Costa ${ }^{1}$ \\ Anne Caroline Coelho Leal Árias Amorim ${ }^{2}$ \\ Aede Gomes Cadaxa ${ }^{3}$ \\ Ana Valéria Machado Mendonça ${ }^{4}$
}

${ }^{1}$ Laboratório de Pesquisa em Comunicação e Saúde, Instituto de Comunicação e Informação Científica

e Tecnologia em Saúde, Fiocruz. Av. Brasil 4365, Manguinhos. 21040-360 Rio de Janeiro RJ Brasil. mariellajornalista@ gmail.com

${ }^{2}$ Departamento de Enfermagem, Universidade de Brasília (UNB). Brasília DF Brasil.

${ }^{3}$ Secretaria de Gestão Estratégica e Participativa, Ministério da Saúde. Brasília DF Brasil.

${ }^{4}$ Departamento de Saúde Coletiva, UNB. Brasília DF Brasil.

\begin{abstract}
The study identifies the discourse and opinions about women's health in relation to their eating habits by means of qualitative research using the journalistic discourse analysis method of the Folha de São Paulo newspaper. Based on the selection of texts on health, women's health and nutrition published throughout the year 2013, the sample resulted in seven journalistic texts, in which voices from the scientific universe related to research, quotes from journals, scientific associations and the opinion of health professionals and researchers were predominantly identified. However, the discourse of women in general about their health rarely appears in the sample analyzed. The main topics were related to health risks and the connection with diet or supplements, as well as pregnancy. The predominant content discourse related to health risk and its relationship with eating habits, nutrition or supplements, as well as pregnancy and/or the manner of delivery; health as a commodity associated with products or procedures, such as diets, dietary supplements, drugs and bariatric surgery, with no room for the promotion of health and healthy eating.

Key words Communication in health, Nutrition, Health promotion, Women's health, Journalism
\end{abstract}

Resumo O estudo identifica discursos e vozes sobre saúde da mulher no que se refere à sua alimentação, por meio de pesquisa qualitativa utilizando o método de análise do discurso jornalístico do impresso Folha de São Paulo. A partir da seleção dos textos sobre saúde, saúde da mulher $e$ alimentação, publicados ao longo do ano de 2013, a amostra resultou em sete textos jornalísticos, nos quais foram identificadas predominantemente vozes provenientes do universo científico relacionadas a pesquisas, citação de periódicos, associações científicas e falas de profissionais da saúde e pesquisadores. Entretanto, as falas de cidadãs comuns pouco apareceram na amostra analisada. Nos conteúdos predominaram o discurso relacionado ao risco à saúde e sua relação com a prática alimentar, nutricional ou de suplementação, como também à gravidez elou ao tipo de parto; a saúde como mercadoria associada a produtos ou procedimentos, como dietas, suplementos alimentares, medicamentos e cirurgia bariátrica, sem espaço para a promoção da saúde e alimentação saudável.

Palavras-chave Comunicação em saúde, Alimentação, Promoção da saúde, Saúde da mulher, Jornalismo 


\section{Introdução}

A mídia de massa pode ser um dos espaços utilizados para promoção da saúde de maneira atrativa para a população, fornecendo informações contextualizada sobre a saúde e seus determinantes e incentivando mudanças de comportamento. Os profissionais da saúde podem utilizar a mídia audiovisual, impressa ou eletrônica para que as mensagens cheguem até a população, seja por meio de campanhas ou artigos de jornal ${ }^{1}$.

A Carta de Otawa já mencionava a mídia como um dos espaços de promoção da saúde, e capacitação da comunidade para atuar na melhoria de sua qualidade de vida, e com maior participação e informação sobre sua saúde. Ou seja, a promoção da saúde não é responsabilidade exclusiva do setor saúde, e o acesso à informação é fundamental para que as pessoas possam tomar decisões que resultem em melhora concreta de saúde ${ }^{2}$. A informação sanitária é um fator produtor de notícias, e na literatura há estudos que analisam desde a difusão de informações de saúde pelos jornalistas ${ }^{3-7}$ até a utilização e apropriação que o público faz das informações que recebe $^{8}$.

A Política Nacional de Promoção da Saúde (PNPS), lançada pelo Ministério da Saúde em 2006 e que em 2014 passou por revisão, apresenta a necessidade de divulgação, sensibilização e mobilização para a promoção da saúde e definiu ações singulares para temas específicos, entre eles a alimentação saudável ${ }^{9-10}$. Sabe-se que as mulheres, além de utilizarem os serviços públicos de saúde mais que os homens ${ }^{11}$, são responsáveis pela escolha dos alimentos de boa parte das famílias. Elas são também alvo certeiro para o marketing da indústria de alimentos; com notável viés de alguém que fala para o público feminino ${ }^{12}$. Sabe-se também que parcela da imprensa brasileira apresenta conteúdos relacionados à saúde da mulher sem qualquer relação com suas necessidades em saúde ${ }^{3}$. Assim, o presente artigo analisa o discurso apresentado em 2013 pelo principal jornal impresso brasileiro, a Folha de São Paulo, nos textos sobre o tema da alimentação e sua interface com a saúde da mulher. Afinal, o periódico colabora para a promoção da saúde das mulheres brasileiras, trazendo informações que contribuam para seu autocuidado e autonomia?

\section{Metodologia}

A amostra compreende os textos publicados pelo jornal impresso Folha de São Paulo ao longo do ano de 2013, acessíveis em formato online por meio de assinatura digital do periódico. A Folha é veículo de comunicação de maior tiragem e circulação entre os diários nacionais de interesse geral (301.299 exemplares da versão impressa), e seu site, possui cerca de 17 milhões de visitantes únicos e 173 milhões de páginas vistas por mês ${ }^{13}$. Os textos foram localizados por meio da palavra chave "saúde". O sentido dado ao termo "saúde" também foi considerado na seleção da amostra. Dessa forma, textos que se referiam a outros contextos, como "saúde do mercado imobiliário" ou "saúde financeira" foram excluídos. Os demais critérios de inclusão dos textos foram: fazer referência à saúde da mulher e à sua alimentação. Por esses critérios foram incluídos na amostra sete textos, alojados em banco de dados do Excel, e organizados segundo o título do texto, dia e mês de publicação.

Para análise foi utilizada a metodologia de análise de discurso ${ }^{14}$ que permite considerar as propriedades discursivas propagadas socialmente, por compreender o ato da linguagem como parte relevante do processo informacional e comunicacional. Os fundamentos que orientam o estudo consideram a informação como discurso e a forma como as mídias agem diante do discurso da informação, considerando: quem informa, para quê, sobre o quê e como informa; e a intencionalidade dos sujeitos de acordo com suas inserções e as posições que assumem diante do mundo em permanente disputa.

Por não se tratar de estudo envolvendo seres humanos, a pesquisa não foi submetida ao Comitê de Ética.

\section{Resultados e discussão}

Considerados os critérios de seleção, 1167 textos sobre saúde foram incluídos na amostra e destes, 107 faziam referência à saúde da mulher, sendo sete deles específicos sobre alimentação, conforme relação do Quadro 1.

Foi aplicada a análise do discurso, à luz da metodologia de Charaudeau ${ }^{14}$, nos sete textos que compõem a amostra. Nestes, foram identificadas as vozes emissoras do discurso e as categorias discursivas que emergiram do texto midiático: quem informa, sobre o quê e como informa e as implicações sociais dos textos jornalísticos 
na comunicação de informações e na construção de compreensões sobre a saúde da mulher e sua alimentação.

\section{Vozes do discurso}

O discurso científico aparece predominantemente nos textos, com recomendações e análises a partir de pesquisas recentes, citação de periódicos, associações científicas e falas de profissionais da saúde e pesquisadores legitimando as afirmações. Obstetrician and Gynaecologist é a única revista científica citada. Também são fontes do textos: pesquisadores de renomadas universidades como Universidade de São Paulo (USP), Universidade Estadual de Campinas (Unicamp) e pesquisas da Santa Casa de São Paulo, Sociedade Brasileira de Cardiologia (SBC) e do Instituto Nacional de Cardiologia (INC).

Os especialistas são os escolhidos pelos jornalistas pois em geral têm conhecimento superior sobre determinado tema em saúde, mas o bom jornalismo não restringe qualquer notícia apenas um tipo de informante, ou fonte. Falar de saúde aliando a voz da ciência a outras, como as de pessoas que vivem determinadas situações, favorece o entendimento dos leitores e torna a informação mais palpável.

Em um dos textos há um contraponto entre uma fonte ligada a empresa de suplementos alimentares e um pesquisador, que contesta o suposto benefício do óleo de coco propagado pela marca, argumentando que não há comprovação sobre seu potencial de emagrecimento. No mesma matéria aparecem os depoimentos das usuárias, ora comprovando o resultado positivo, ora apresentando efeitos colaterais negativos com o uso. Considerando-se que cada sujeito é único e que a saúde incita uma visão complexa, percebese que tal diálogo pode qualificar a compreensão do leitor sobre a apropriação das indicações relatadas para si.

As usuárias que divulgam e vendem sua dieta ganham voz em outro texto que mostra os benefícios da personal trainer que virou vendedora e de uma advogada que lucra com um blog sobre dieta. Sem qualquer contraponto ou outro lado, o texto apresenta a mudança de alimentação como causa do emagrecimento e mostra os benefícios de se investir em propagar a dieta, sem ponderar as especificidades de cada mulher, os benefícios da alimentação saudável e os riscos de dietas, como os conselhos comercializados por leigos sem o acompanhamento de profissional da área de nutrição ou outro, ligado à área de saúde. As mulheres são as principais consumidoras de temas de saúde na imprensa ${ }^{15}$, mas as falas de cidadãs comuns pouco aparecem na amostra analisada, e quando estão nos textos, são ancoradas no uso de artifícios externos para perder peso, sem citar os benefícios da alimentação saudável ou os riscos relacionados às dietas alimentares. Trechos como "é bem mais fácil só tomar um comprimido" e "as coisas começaram a mudar só com a internação", ilustram essa mulher à mercê de uma técnica ou medicação salvadora de seu peso e não necessariamente empoderada para a promoção de sua saúde. Em outro texto, o argumento da cidadã sobre os benefícios de se tomar determinado óleo para emagrecer é desqualificado pelo profissional de saúde. "Em excesso, vai engordar e fazer mal".

Da mesma forma, nas falas dos profissionais, a necessidade de mudar hábitos para uma alimentação saudável também não aparece explicitamente, mas como um não dito, quando por exemplo se afirma que "não adianta só dar o remédio. Precisa atingir a meta”. Mas não se explica se a meta é a saúde, ou só a redução do colesterol, deixando a informação vaga para o leitor. Considerando-se que boa parte das pessoas tem a 
imprensa como única fonte de conhecimento em saúde ${ }^{16}$, informações vagas não contribuem para a autonomia delas na tomada de decisão sobre sua saúde.

\section{Risco Onipresente}

$\mathrm{Na}$ maioria dos textos jornalísticos analisados, identificou-se a presença do discurso sobre o risco à saúde e a sua relação com a prática alimentar, nutricional ou de suplementação. Aqueles relacionados ao estilo de vida aparecem com maior destaque, seja o risco relacionado à dieta inadequada, que gera problemas de saúde como a obesidade e o colesterol alto ou aquele associado às dietas que têm benefício questionável, como no caso da matéria sobre a eficácia do uso dos óleos vegetais para emagrecer; ou mesmo os textos que relacionavam o risco da obesidade à gravidez e ao parto, como nas matérias "Reduzir o estômago eleva risco na gravidez", "Cesárea pode ser causa de obesidade durante vida adulta"e "Precisei ficar 'presa' no hospital para parar de comer”. Esta última relata a história de uma dona de casa obesa que engordou gradativamente após cada gravidez.

Essa onipresença do discurso do risco nas matérias jornalísticas reproduz o status atual que esse discurso ocupa em nosso tempo. A sociedade de controle é terreno propício para dissociação da "ciência das doenças" da "arte de curar" ditada pela medicina científica. Illich ${ }^{17}$ analisa essas mudanças por que passam as sociedades ocidentais, a partir do tema da autonomia dos indivíduos e dos modos de interpretação e ação frente aos seus problemas de saúde ${ }^{18}$.

A temática da gestão de riscos, seja para identificá-los ou reduzi-los, tornou-se um objetivo central da saúde pública, que constrói sentidos baseados na racionalidade da ciência e seus sistemas "de peritos de conhecimento"19-21, sendo tais experts que passam a ditar a forma como conduzir nossa saúde e estilo de vida, mediante gestão da totalidade das informações sobre os riscos estudados, medidos e nomeados ${ }^{22}$.

E é via comunicação do risco, feita em grande medida pelos meios de comunicação de mas$\mathrm{sa}^{23}$, que se reforça a retórica do risco voltada ao estilo de vida e do seu impacto na promoção da saúde ${ }^{22-20}$, como identificado nas matérias analisadas, cujo discurso relaciona práticas inerentes à vida, como alimentar-se ou engravidar a um comportamento de risco ou desvio.

Assim, condutas do cotidiano muitas vezes passam a ser vistas como perigosas, a partir da interpretação epidemiológica apresentada pela mídia, em especial por meio do jornalismo, um dos responsáveis por traduzir e legitimar aos leigos o conhecimento dos experts ${ }^{23,24}$.

De certa forma, jornalistas exercem por meio das rotinas midiáticas de enquadramento e agendamento da notícia, uma pedagogia da vida saudável a partir da divulgação de estudos científicos que relacionam os riscos para saúde ${ }^{24}$. Seja na tradução das informações dos experts, como revelado na matéria que destaca as novas diretrizes da SBC para o controle dos níveis de colesterol entre mulheres; seja a que constrói categorias e grupos de risco, como o texto que aponta a gravidez isoladamente pode se tornar um risco para obesidade:

pesava $63 \mathrm{~kg}$ aos 14 anos, quando engravidou da primeira filha. Ao final da gestação, atingiu os $100 \mathrm{~kg}$ e não parou mais [...] tive minha segunda filha [...] e fui para uns $130 \mathrm{~kg}$. Com 22 anos, tive a [...] e fui para $163 \mathrm{~kg}$ [...] Depois da gestação, ganhei mais $20 \mathrm{~kg}$.

Essa medicalização da sociedade vem assumindo novas expressões que encontram alicerce nas distorções da promoção da saúde, quando o controle da ciência médica passa para além do doente e é reforçado por meio dos conselhos e cuidados que devem ser dispensados às pessoas em perfeita saúde, a fim de controlar seu estilo de vida e com isso reduzir os possíveis riscos ${ }^{19}$. Nesse sentido, de seres viventes que se alimentam, passamos à pacientes, que controlam a dieta para evitar riscos cardiovasculares provocados pela ingestão de gorduras, ou às pessoas que consomem produtos específicos, como os óleos vegetais, a fim se manterem magros para com isso obter ou manter a qualidade de vida.

\section{Pílulas Mágicas}

Essa visão da vida medicalizada e da saúde como mercadoria é estruturante para construção do discurso presente em outra categoria identificada nos textos analisados: a pílula mágica que vende benefícios de uma boa saúde, como a perda de peso de forma rápida e fácil, por meio de uma ação de consumo. Trecho de umas das matérias que entrevista uma consumidora de medicamentos para emagrecer exemplifica muito bem essa categoria discursiva: "Agora estou controlando o peso com dieta, mas está muito mais difícil. É bem mais fácil só tomar um comprimido.”

Formas mais dolorosas e arriscadas, como no caso da cirurgia bariátrica, também são vistas como mercadoria. Um bem de consumo que per- 
mite alcançar o estilo de vida pretendido, ou seja, perda de peso=vida saudável=qualidade de vida, como revelado no trecho do depoimento de uma obesa internada a espera de cirurgia bariátrica: "Meu primeiro sonho depois da cirurgia é colocar um tênis. Há mais de dez anos não uso tênis, só chinelo (...) Quando sair daqui, vou ser uma nova mulher, uma nova mãe, uma nova esposa".

Assim, o que pode ser fornecido e consumido toma o lugar do que pode ser feito pela mulher, de maneira autônoma, promotora de autocuidado e redutora de danos, o que poderia prevenir mortes oriundas de práticas desnecessárias e que alicerçam uma saúde como "bem de mercado". Essa visão reflete interesses pouco ou nada defensores da vida, como explicitam os trechos "usuárias de redes sociais que compartilham na web a rotina de seus regimes faturam até $\mathrm{R} \$ 10 \mathrm{mil}$ por mês com suas receitas de saúde e emagrecimento", "procurei um endócrino e disse que meu objetivo era emagrecer. Comecei a tomar a sibutramina e o efeito foi imediato". Chama a atenção que um dos textos analisados aponta para os reflexos dessa cultura medicalizante: "além de mais mulheres entrarem no perfil de alto risco, as metas máximas de colesterol (...) vão mudar (...). A consequência é clara: mais pessoas devem receber a indicação de tomar remédios para baixar o colesterol, as estatinas". Esses dados já foram confirmados em congresso recente da Associação Latino Americana de Ciências da Comunicação (ALAIC), no qual apresentou-se o discurso produzido em 2011 sobre os emagrecedores em quatro revistas semanais brasileiras contrários a dimensão defensora da promoção da saúde e que valorizam a culpabilização das pessoas e o aprofundamento da distância entre gestores, profissionais da saúde e cidadãos ${ }^{25}$. De acordo com esse estudo, os textos não conceituam adequadamente os riscos, omitem as controvérsias do campo científico e político e adotam como verdade absoluta apenas um lado da questão. Uma vida saudável aparece como responsabilidade única do indivíduo e a interpretação e manejo de dados epidemiológicos é relatado como algo restrito aos órgãos reguladores, governo e profissionais de saúde, desconsiderando as especificidades de cada sujeito.

Portanto, o discurso dos textos jornalísticos analisados na Folha de São Paulo ao associarem informações de origem científica sobre alimentação, especialmente dietas e procedimentos médicos, a exemplos de beleza, saúde e estilo de vida constroem ideais e estereótipos de saúde feminina com ênfase nas soluções instantâneas, de con- cepção biológica, e as associa ao consumismo de bens e serviços provenientes das indústrias médica e de alimentação e nutrição, como no caso da cirurgia bariátrica, dos medicamentos para emagrecer, das dietas e dos suplementos da moda.

\section{Militarismo do peso: desafios na formação do profissional de saúde e autonomia da mulher}

O uso de medicações e suplementos alimentares para emagrecer tem sido comum na atualidade. Entretanto, muitas mulheres que o fazem não têm, em muitos casos, o amplo conhecimento dos riscos ligados ao controle do peso oriunda das vias medicamentosa e de suplementação alimentar. Os padrões de beleza ao longo da história têm levado as mulheres, em particular, a fazer dietas variadas sem muito conhecimento de riscos e benefícios. $\mathrm{Na}$ análise dos discursos percebeu-se expressões contraditórias acerca do uso de sibutramina com a finalidade de controlar o peso.

Trechos discursivos da reportagem intitulada "Você recupera o peso com a mesma facilidade com que perde" revela tal dicotomia, que embora refira à resposta medicamentosa imediata, apresenta os efeitos colaterais e a replicação do problema de saúde pela paciente, como a omissão dos riscos e as determinações:

procurei um endócrino [...] meu objetivo era emagrecer. Comecei a tomar a sibutramina e o efeito foi imediato [...] Não sentia fome, só dor de cabeça [...] gosto de metal na boca. [...] a perda foi ficando mais lenta, e desisti [...] estava com a 'boca solta' e engordei bastante.

Outra médica me receitou femproporex [...] senti mais resultado que com a sibutramina. Mas parei e voltei a engordar. Depois, a Anvisa proibiu [...] só podia me receitar sibutramina. Tomei e emagreci $3 \mathrm{~kg} \mathrm{em}$ um mês. Resolvi parar de vez porque li sobre os efeitos colaterais e tenho histórico familiar de doenças cardíacas. Não quero depender de remédio para emagrecer para sempre.

Esses trechos permitem refletir sobre como a formação em saúde reflete um despreparo profissional quanto ao aspecto informacional promotor de autocuidado e quanto à necessidade de aderir-se às tecnologias de informação e comunicação em saúde (TICS) como meios de empoderar a população e alertá-la para os interesses mercantis e os riscos a eles relacionados ${ }^{14,26}$. Corroborando com a discussão, a Política Nacional de Humanização ${ }^{27}$ ressalta a autonomia dos sujeitos, mobilizando-os a tornarem-se protagonis- 
tas de sua saúde. Os profissionais de saúde por terem maior conhecimento dos determinantes do processo de adoecimento, especialmente os do primeiro nível de atenção, podem potencializar essa autonomia fornecendo informações que promovam uma maior capacidade de resposta ${ }^{28}$ por parte dos usuários do Sistema Único de Saúde, e induzam esses usuários a corresponsabilizarem-se por mudanças comportamentais centrais em sua saúde individual e coletiva ${ }^{29,30}$.

A reportagem sobre o efeito emagrecedor do óleo de coco, apresenta como verdade a prévia de resultados de outra pesquisa e dialoga com trechos de um blog, que divulga o questionável efeito "redutor de peso", o qual não tem evidências científicas, isoladamente, podendo provocar aumento do peso e distúrbios hidro-eletrolíticos com seu uso, como descritos nas falas: "o óleo de coco, sozinho, engorda. Não há milagre", esclarece a nutricionista; "deu tudo errado (...) tive diarreia e ânsia (...) se emagreci foi de tanto passar mal", referiu a paciente.

Outro tema abordado relaciona a obesidade futura à opção pela cesariana como via de parto e consequente baixo peso ao nascer, na hipótese de que a microbiota do corpo da criança seria alterada no parto cesáreo e que tal alteração levaria a mudanças fisiológicas e do metabolismo infantil, capazes de favorecer o desenvolvimento de obesidade na vida adulta. Há desafios atuais sobre o ensino da área médica, o qual sofre pressões e conflitos éticos, que podem levar os profissionais da saúde a estimular as cesariana entre as mulheres. A instituição formadora pode não ter como assegurar a todos os estudantes oportunidades destes fazerem partos normais, mas, sobretudo, o lucro que esse profissional terá ou que perderá com sua opção clínica (cesárea ou parto normal), ou seja, o que é evidenciado no texto jornalístico quando se diz o parto normal "pode levar oito a dez horas", e "pode fazer com que o médico perca as consultas marcadas no dia", ao passo que se ele optar pelo parto natural há redução do seu lucro quando o discurso infere que "ele não ganha por isso".

De forma convergente, a reportagem " $\mathrm{Mu}$ lheres terão limites mais rígidos para colesterol 'ruim" reforça o duplo interesse da área médica e farmacêutica que atrela pacientes com risco cardiovascular a medidas mais medicamentosas e menos promotoras de saúde. Tal constatação ocorre por que embora as diretrizes da SBC apontem que o uso da medicação reduz o risco de problemas como infarto, derrame e insuficiência cardíaca, o texto jornalístico deixa claro que as estatinas têm participação terapêutica, questionável, nos tratamentos da colesterolemia e que mais que medicalizar, a classe médica teria que sair da "inércia terapêutica", pois o texto jornalístico traz a informação de que "hoje a maioria dos que tomam estatina tem uma redução pequena do HDL". Embora nesses trechos jornalísticos se evidencie o efeito redutor de danos cardiovasculares das estatinas, percebe-se a necessidade de trazer vozes nas reportagens que apresentem alternativas promotoras de saúde, ao passo que reforçam os interesses do mercado mantenedor da doença. Então, a promoção da saúde tornase ainda mais valorosa para mulheres e toda a população, e reacende a visão de que a atividade física, aliada a qualidade alimentar, tem maior potencial para favorecer a saúde, entretanto, recomendações como essas não dão lucro à classe médica, nem à indústria farmacêutica e, por isso, podem estar sendo excluídas das coberturas jornalísticas.

\section{Considerações finais}

As informações que circulam na imprensa, sobre saúde da mulher e alimentação não são isentas e estão atreladas a compromissos e interesses de diferentes atores e dos sentidos atribuídos por eles à saúde e qualidade de vida feminina e sua relação com alimentação e o controle do peso. Nos textos analisados percebeu-se a linguagem de armadilhas, que oriunda da polidiscursividade ${ }^{14}$ revela interesses inter-relacionados da ciência, das indústrias médico-farmacêutica e de alimentação e nutrição, como dos profissionais de saúde e das mulheres, em menor grau.

Nesse contexto, a importância da imprensa se mostra principalmente pela circulação de ideias que influenciam no cotidiano de grande faixa da população, pois esta amplia os discursos e possibilita-lhes tanto a eleição de novos sentidos quanto a desqualificação de outros, como a construção de versões diversas sobre alimentação, controle de peso e saúde.

A construção dos discursos se divide em vozes predominantes de experts da área de saúde, mas pouco asseguradas por veículos de informação científica; de certa maneira as reportagens evidenciam sobretudo os aspectos relativos à visão 'patogênico-centrada'e poucas são as iniciativas profissionais originalmente salutogênicas.

A medicalização e o risco são centrais nos discursos das matérias, o que reforça a necessidade de revisão das instituições formadoras quanto à 
capacidade crítico-reflexiva, sobretudo dos médicos, em relação ao uso de medicamentos como medida central da ação de saúde e imprime à academia maior responsabilidade quanto ao desafio de formar sujeitos capazes de tornar o usuário partícipe de sua saúde, em superação ou controle da doença, e que o cuidado seja a base de sua prática, não o lucro.

\section{Colaboradores}

MS Oliveira-Costa participou na concepção, delineamento, análise, interpretação dos dados e redação do artigo, ACCLA Amorim participou na análise e redação, AG Cadaxa participou na análise, interpretação dos dados e redação, AVM Mendonça participou da análise e revisão crítica da versão publicada.

\section{Agradecimentos}

Aos auxiliares de pesquisa estudantes de Saúde Coletiva da Universidade de Brasília que participaram da coleta de dados Mariane Leonel, Bárbara Gonçalves, João Armando Alves, Rodrigo Silvério e Madga Saraiva.

\section{Referências}

1. Corcorán N, organizador. Comunicação em saúde. Estratégias para promoção da saúde. São Paulo: Roca; 2010.

2. Brasil. Ministério da Saúde (MS). Secretaria de Políticas de Saúde. Projeto Promoção da Saúde. As Cartas da Promoção da Saúde. Brasília: MS; 2002.

3. Oliveira MS, Paiva LHC, Costa JV, Pinto-Neto AM. Imprensa e Saúde da mulher: a abordagem das revistas semanais brasileiras. Rev Brasileira de Ciências da Comunicação 2009; 32(1):109-128.

4. Oliveira VC. Os sentidos da saúde nas mídias jornalísticas impressas. RECIIS, Brasil, 6, feb. 2013. [acessado 2013 maio 1]. Disponível em: http://www.reciis.cict. fiocruz.br/index.php/reciis/article/view/731/1368

5. Schwartz L, Woloshin S, Andrews A, Stukel T. Influence of medical journal press releases on the quality of associated newspaper coverage: retrospective cohort study. BMJ 2012; 344. [acessado 2013 maio 1]. Disponível em: http://www.bmj.com/content/344/bmj.d8164

6. Chagas C, Massarani L, Ramalho M, Reznik G. Investigação em medicina e saúde no horário nobre: análise de dois programas televisivos brasileiros. $\mathrm{Ra}$ zón y Palabra 2013; 82(mar-maio). [acessado 2013 maio 10]. Disponível em: http://www.redalyc.org/ pdf/1995/199525737016.pdf

7. Massarani L, Chagas C, Ramalho M, Reznik G. Saúde aos domingos' uma análise da cobertura da pesquisa em medicina saúde no Fantástico - RECIIS, Brasil, 7, mar. 2013. [acessado 2013 maio 11]. Disponível em: http://www.reciis.cict.fiocruz.br/index.php/reciis/article/view/706/1451

8. Renaud L. Les medias et la santé: de l'emergence àl'appropriation des normes sociales. Quebec: Presses de LÚniversitédu Québec; 2010. [acessado 2014 jun 3]. Disponível em: http:/comsante.uqam.ca/publications/ livres/42-les-medias-et-la-sante-de-lemergence.html

9. Brasil. Ministério da Saúde (MS). Secretaria de Vigilância em Saúde. Política nacional de promoção da saúde. Brasília: MS; 2006.

10. Brasil. Ministério da Saúde. Portaria No 2.446, de 11 de novembro de 2014. Redefine a Política Nacional de Promoção da Saúde (PNPS). Diário Oficial da União 2014; 12 nov. 
11. Ruiz G. Quem usa o Sistema Único de Saúde? Rio de Janeiro: Portal DSS Brasil; 2012 Abr 09. [acessado 2013 nov 15]. Disponível em: http://dssbr.org/site $/$ ? $=9534 \&$ preview $=$ true

12. Lacaille E. Cacophonie alimentaire et les femmes québécoises: des modeles alimentaire en mutation. Montreal: Universidad du Quebéc à Montreal; 2009.

13. Folha de São Paulo. Conheça a Folha de São Paulo. [acessado 2014 maio 10]. Disponível em: http://wwwl. folha.uol.com.br/institucional/circulacao.shtml

14. Charaudeau P. Discurso das mídias. São Paulo: Contexto; 2012.

15. Tabakman R. A saúde na mídia: medicina para jornalistas, jornalismo para médicos. São Paulo: Summus Editorial; 2013.

16. Mosquera M. Comunicación en salud: conceptos, teorías y experiencias. Comminit, La iniciativa de la comunicación, 2003. [acessado 2013 mar 27]. Disponível em: http://www.comminit.com/en/node/150400

17. Illich I. A expropriação da saúde: nêmesis da medicina. $4^{a}$ ed. São Paulo: Nova Fronteira; 1975.

18. Tesser CD. Medicalização social (I): o excessivo sucesso do epistemicídio moderno na saúde. Interface (Botucatu) 2006; 10(19):61-76.

19. Azevedo E. Reflexões sobre riscos e o papel da ciência na construção do conceito de alimentação saudável. Rev. Nutr. 2008; 21(6):717-723. [acessado 2014 nov 23]. Disponível em: http://www.scielo.br/scielo.php?script =sci_arttext\&pid=S1415=52732008000600010-\&lngen\&nrm =iso

20. Neves TP, Guilam MCR. Diminuindo riscos, promovendo vida saudável: o conceito de risco na promoção da saúde. Salusvita 2007; 27(3):283-299.

21. Carvalho SR. As contradições da promoção àsaúde em relação àprodução de sujeitos e a mudança social. Cien Saude Colet 2004; 9(3):669-678.

22. Bagrichevsky M, Castiel LD, Vasconcellos-Silva PR, Estevão A. Discursos sobre comportamento de risco à saúde e a moralização da vida cotidiana. Cien Saude Colet 2010; 15(Supl. 1):1473-1482.

23. Rangel SM. Comunicação no controle de risco à saúde e segurança na sociedade contemporânea: uma abordagem interdisciplinar. Cien Saude Colet 2007; 12(5):1375-1385.

24. Castiel LDM, Vasconcellos-Silva PR. Precariedade do excesso: informação e comunicação em saúde coletiva. Rio de Janeiro: Fiocruz; 2006.
25. Amaral VM, Laguardia J. Mídia e risco a saúde: o caso dos emagrecedores nas revistas semanais de informação. Congresso Alaic 2014. [acessado 2014 set 10]. Disponível em: http://congreso.pucp.edu.pe/alaic2014/ wp-content/uploads/2013/09/Vanessa-Amaral.pdf

26. Wolton D. Informar não é comunicar. Porto Alegre: Sulina; 2011.

27. Brasil. Ministério da Saúde (MS). Secretaria Executiva. Núcleo Técnico da Política Nacional de Humanização. Documento Base. Brasília: Editora MS; 2008.

28. Santos PNV, Assis MMA, Amorim ACCA, Abreu de Jesus WL, Santos AM, Rodrigues AAAO. Construção política e simbólica da participação dos sujeitos na concretização do acesso àEstratégia Saúde da Família. In: Assis MMAA, Almeida MGV, organizadores. Acesso aos serviços e tecnologias no Sistema Único de Saúde: abordagens teóricas e práticas. Feira de Santana: UEFS Editora; 2014. p. 291-328. v.1

29. Amorim ACCLA, Lima PVSF, Silva PFS, Silva KNC, Ferreira MPF, Sárc. Considerações sobre internação domiciliar e a inserção da família no cuidado de Enfermagem: relato de caso. Gestão e Saúde 2014; 5(edição especial). [acessado 2014 nov 27]. Disponível em: http://www.gestaoesaude.unb.br/index.php/gestaoesaude/issue/view/EDI\%C3\%87\%C3\%83O\%20ESPECIAL/showToc

30. Lima PVSF, Nascimento AKC, Seganfredo HC, Ribeiro IC, Amorim ACCLA, Alves ED. Processo de Enfermagem, segundo o modelo conceitual de Wanda Horta, aplicado a uma família atendida pelo Núcleo Regional de Atenção Domiciliar, Distrito Federal. Gestão e Saúde 2014; 5(2). [acessado 2014 nov 27]. Disponível em: http://gestaoesaude.unb.br/index.php/gestaoesaude/ article/view/795

Artigo apresentado em 02/03/2015

Aprovado em 22/08/2015

Versão final apresentada em 24/08/2015 